\title{
Los indicadores financieros y el Valor Económico Agregado (EVA) en la creación de valor
}

(1) Jorge Vergiú Canto (2) Christian Bendezú Mejía

\section{RESUMEN}

La meta de cualquier empresa es buscar elevar el valor de la empresa a través de maximizar las utilidades para los accionistas, equilibrar el pasivo y el patrimonio a fin minimizar los niveles de riesgo y tener una liquidez adecuada para afrontar los compromisos del corto plazo Es en esta búsqueda que se comienza el verdadero reto de hallar el valor de la empresa e interpretarla, para lo cual se tiene dos planteamientos: el tradicional a través de los Indicadores Financieros que si bien son razones claras y fáciles de calcular, reducen su efectividad como instrumentos de medición de riqueza y el Valor Económico Agregado (EVA) el cual es una herramienta que trata de contrarrestar las limitaciones de estos indicadores al valorizar y evaluar el desempeño de la gestión a través de la creación de valor.

Palabras Clave: Indicadores financieros, Bolsa de Valores, Valor Económico Agregado rentabilidad, accionistas.

FINANCIAL INDICATORS AND ADDED ECONOMIC VALUE IN VALUE CREATION ABSTRACT

An enterprise aim is to search the increasing of an enterprise's value through maximizing utilities for shareholders, balancing liabilities and heritage with the purpose of minimizing risk levels and thus to obtain a suitable solvency in order to face short-time engagements. The real challenge to find the enterprise's value and to interpret it, through this search, is that, for that reason, two approaches are made: the traditional one, through Financial Indicators that, even though they are considered to be clear and easy reasons to be worked out, might decrease their effectiveness as instruments for wealth measuring and the Added Economic Value (AEV) which is a tool which tries to counteract these indicators limitations by valueing and assessing any management performance through value creation.

Key Words: Financial indicators, stock exchange, Added Economic Value, profitability, shareholders.

\section{INTRODUCCIÓN}

Actualmente las empresas se valorizan no sólo por sus activos sino también por sus marcas, desarrollo de nuevas tecnologías, su porción en el mercado y la creatividad. Conceptos de activos intangibles que la contabilidad tradicional no contempla en el registro de movimientos económicos para la determinación de los estados financieros, de ello se desprende la inquietud como valorizar realmente a la empresa, esto se puede realizar a través del Valor Económico Agregado (EVA), el cual es un indicador económico cuyo objetivo básico es medir el resultado de la gestión en el negocio.

\section{CONCEPTOS GENERALES}

\section{Costo de Capital:}

Una empresa para financiar sus necesidades de capital puede acudir a dos fuentes básicas de recursos financieros: deuda y capital propio. La deuda es capital de terceros (en especial proveedores y entidades financieras), y el capital propio que es el aporte de los inversionistas, accionistas o socios de la empresa. Ambos recursos tienen un costo para la empresa, el cual se puede expresar en términos de tasa de interés. (1)

\section{Costo Promedio Ponderado del Capital (CPPC)}

Este valor es un dato fundamental para el proceso de toma de decisiones de inversión. El cálculo se realiza multiplicando el costo específico de cada forma de financiamiento por su proporción en la estructura de capital de la empresa y sumando los valores ponderados. (1)

$$
\mathrm{CPPC}=\mathrm{Kd} \times(\text { pasivo/activo }) \times(1-\mathrm{t})+\mathrm{Kc} \times(\text { patrimonio / activo })
$$

Kd: Costo de la Deuda.

Kc: Costo de Capital Propio.

Mediante estos ratios financieros los gerentes financieros, las financieras que otorgan créditos de diversa índole y los analistas de mercado de capitales buscan determinar qué factores del negocio están afectando a la empresa en su crecimiento. Pero este análisis presenta algunas desventajas como:

- Grandes empresas tienen diversas líneas productivas que no necesariamente están dentro un sector económico por lo que es difícil encontrar un promedio para compararlas con otras empresas.

\footnotetext{
(1) Ingeniero Industrial, Profesor del Departamento de Producción y Gestión Industrial UNMSM

E-mail : jvergiuc@yahoo.com

E-mail : christian_bendezu@yahoo.com
} 


\section{Cuadro 1. Indicadores Financieros para la medición de la creación de valor (2)}

\begin{tabular}{|c|c|c|}
\hline $\begin{array}{l}\text { Precio de mercado de } \\
\text { las acciones }\end{array}$ & \multicolumn{2}{|c|}{$\begin{array}{l}\text { La creación de valor tradicional para el accionista es analizar la evolución del } \\
\text { precio del mercado de las acciones de la empresa. Este indicador es entendible y } \\
\text { fácil de obtener pero la evolución no depende necesariamente de la empresa } \\
\text { sino del movimiento del mercado que en muchos caso s no está de acuerdo a la } \\
\text { actuación empresarial. }\end{array}$} \\
\hline $\begin{array}{l}\text { Rentabilidad } \\
\text { Empresarial }\end{array}$ & $\begin{array}{l}\text { Este indicador mide el éxito alcanzado } \\
\text { por la empresa. Permite tener en } \\
\text { cuenta la utilidad generada por cada } \\
\text { unidad de negocio. } \\
\text { Entre sus limitaciones se tiene los } \\
\text { criterios contables aplicados entre } \\
\text { empresa difieren y los impactos } \\
\text { futuros que se pudieran dar al evaluar } \\
\text { estados financieros finalizados }\end{array}$ & $\frac{\text { Utilidad Neta }}{\text { Ventas }}$ \\
\hline Utilidad por Acción & $\begin{array}{l}\text { Tiene una limitación adicional de que } \\
\text { la utilidad por acción no puede ser } \\
\text { calculada por unidad de negocio. }\end{array}$ & $\frac{\text { Utilidad Neta }}{\text { Número de Acciones }}$ \\
\hline $\begin{array}{l}\text { Flujo de caja por } \\
\text { Acción }\end{array}$ & $\begin{array}{l}\text { Se calcula a partir de una } \\
\text { aproximación de la tesorería. } \\
\text { Teniendo las mismas limitaciones de } \\
\text { la utilidad por acción. }\end{array}$ & $\frac{\text { Utilidad Neta }+ \text { Depreciaciones }}{\text { Número de Acciones }}$ \\
\hline $\begin{array}{l}\text { Rentabilidad del Activo } \\
\text { (ROI) }\end{array}$ & $\begin{array}{l}\text { La rentabilidad del activo o ROI (del } \\
\text { inglés Return on Investment ) es un } \\
\text { indicador muy utilizado en la } \\
\text { evaluación de empresas. } \\
\text { La financiación pasa a segundo plano } \\
\text { y no se considera el costo del dinero ni } \\
\text { el nivel de riesgo con la que opera la } \\
\text { empresa. }\end{array}$ & $\frac{\text { Utilidad antes de Int. e Imp. }}{\text { Activo }}$ \\
\hline
\end{tabular}

- La estacionalidad: muchas empresas tienen periodos en los cuales las ventas se incrementan (como en el sector confecciones: día de la madre, fiestas patrias, fiestas navideñas y año nuevo).

- La inflación: los valores de los estados financieros cambian debido a la actualización de valores

- Diferentes prácticas contables entre empresas dentro del rubro y por ultimo la dificultad de establecer una escenario estándar en la cual se pueda dar la certeza si es que una empresa es buena a través de los ratios.

En resumen estos ratios tiene limitaciones como medio para la medición de la riqueza creada para el accionista, estos consideran variables decisivas como el costo del capital, el riesgo en el que opera la empresa y expectativas del futuro.

Valor Económico Agregado (EVA) (3)/(4)

El EVA mide si la utilidad es suficiente para cubrir el Costo de Capital empleado en la generación de utilidad. Su resultado les da a los accionistas, inversionistas e interesados elementos de juicio para visualizar si se generó valor en determinado periodo de tiempo.

En texto del CPC Mario Apaza Meza menciona que Peter Drucker comento: "El EVA está basado en algo que hemos sabido por largo tiempo: aquello que en los estado financieros denominados utilidades, usualmente no representan verdaderas utilidades. Hasta que un negocio no genere una utilidad superior al costo de capital...."

Deduciendo la fórmula esta se puede presentar de la siguiente forma:

\begin{tabular}{l|l|}
\hline EVA $=$ & ROI $\times$ Capital $-K c \times$ Capital \\
\hline EVA $=$ & $($ ROI $-K c) \times$ Capital \\
\hline
\end{tabular}

Donde:

\begin{tabular}{|lr|l|}
\hline ROI & $=$ & Retorno sobre la inversión del capital \\
\hline Kc & $=$ & Costo de Oportunidad \\
\hline Capital & $=$ & Capital Empleado \\
\hline
\end{tabular}

Como se puede analizar lo que se busca a través del EVA es definir cuanta rentabilidad deberá recibir el empresario por el capital empleado, esta debe compensar el riesgo tomado por estar en un determinado negocio. De no presentarse una diferencia positiva la empresa estaría operando en pérdida. El EVA o utilidad económica se fundamenta en que los recursos empleados por una empresa o unidad estratégica de negocio debe producir una 
Cuadro 2. Estados financieros Alicorp. (05)

\begin{tabular}{|c|c|c|}
\hline \multicolumn{3}{|l|}{ ACTIVO } \\
\hline Activo Corriente & 2005 & 2004 \\
\hline Caja y Bancos & 11,069 & 5,420 \\
\hline Valores Negociables (neto de provisión acumulada) & 0 & $\underline{0}$ \\
\hline Cuentas por Cobrar Comerciales (neto de provisión acumulada) & 253,450 & 258,608 \\
\hline Cuentas por Cobrar Vinculadas & 12,663 & 30,391 \\
\hline Otras Cuentas por Cobrar (neto de provisión acumulada) & 98,822 & 100,434 \\
\hline Existencias (neto de provisión acumulada) & 273,645 & 230,731 \\
\hline Activos por Instrumentos Financieros Derivados & 3,288 & $\underline{0}$ \\
\hline Gastos Pagados por Anticipado & 16,572 & 14,687 \\
\hline Total Activo Corriente & 669,509 & 640,271 \\
\hline \multicolumn{3}{|l|}{ Activo No Corriente } \\
\hline Cuentas por cobrar comerciales a largo plazo & 0 & 0 \\
\hline Cuentas por Cobrar a Vinculadas a Largo Plazo & 1,637 & 3,235 \\
\hline Otras Cuentas por Cobrar a Largo Plazo & 0 & 0 \\
\hline Existencias & 0 & 0 \\
\hline Inversiones Permanentes (neto de provisión acumulada) & 73,971 & 59,216 \\
\hline Activos por Instrumentos Financieros Derivados & 0 & 0 \\
\hline Inversiones en Inmuebles & 0 & 0 \\
\hline Inmuebles, Maquinaria y Equipo Neto & 793,626 & 833,082 \\
\hline Activos Intangibles Neto & 99,069 & 28,476 \\
\hline Impuesto a la Renta y Participaciones Diferidos Activo & 1,301 & 1,266 \\
\hline Crédito Mercantil & 105,766 & 105,766 \\
\hline Otros Activos & 72,261 & 91,130 \\
\hline Total Activo No Corriente & $1,147,631$ & $1,122,171$ \\
\hline TOTAL ACTIVO & $1,817,140$ & $1,762,442$ \\
\hline \multirow{2}{*}{\multicolumn{3}{|c|}{ PASIVO + PATRIMONIO }} \\
\hline & & \\
\hline Sobregiros Bancarios & 6,073 & 5,695 \\
\hline Préstamos Bancarios & 204,417 & 177,041 \\
\hline Cuentas por Pagar Comerciales & 152,097 & 199,026 \\
\hline Cuentas por Pagar a Vinculadas & 1,083 & 929 \\
\hline Otras Cuentas por Pagar & 67,368 & 39,054 \\
\hline Parte Corriente de las Deudas a Largo Plazo & 68,875 & 37,161 \\
\hline Pasivos por Instrumentos Financieros Derivados & 0 & 10,125 \\
\hline Total Pasivo Corriente & 499,913 & 469,031 \\
\hline \multicolumn{3}{|l|}{ Pasivo No Corriente } \\
\hline Deudas a largo plazo & 223,991 & 280,831 \\
\hline Cuentas por pagar a vinculadas & 0 & 0 \\
\hline Pasivos por Instrumentos Financieros Derivados & 0 & 0 \\
\hline Ingresos Diferidos (netos) & 943 & 1,089 \\
\hline Impuesto a la Renta y Particip.Diferidos Pasivo & 54,234 & 52,991 \\
\hline Total Pasivo No Corriente & 279,168 & 334,911 \\
\hline Total Pasivo & 779,081 & 803,942 \\
\hline Contingencias (Solo debe mostrarse cuando exista) & 0 & 0 \\
\hline Interés minoritario & 0 & 0 \\
\hline \multicolumn{3}{|l|}{ Patrimonio Neto } \\
\hline Capital & 847,192 & 847,192 \\
\hline Capital adicional & 0 & 0 \\
\hline Acciones de Inversión & 7,388 & 7,388 \\
\hline Resultados no realizados & $-83,367$ & $-77,300$ \\
\hline Excedente de Revaluación & 37,206 & 37,206 \\
\hline Reservas Legales & 6,679 & 0 \\
\hline Otras Reservas & 24 & 24 \\
\hline Resultados Acumulados & 222,937 & 143,990 \\
\hline Efecto acumulado por reexpresión a moneda extranjera & 0 & 0 \\
\hline Total Patrimonio Neto & $1,038,059$ & 958,500 \\
\hline TOTAL PASIVO Y PATRIMONIO NETO & $1,817,140$ & $1,762,442$ \\
\hline
\end{tabular}




\section{Cuadro 3. Estados de Pérdidas y Ganancias}

\begin{tabular}{|c|c|c|}
\hline Ingresos Operacionales & 2005 & 2004 \\
\hline Ventas Netas (ingresos operacionales) & $1,817,782$ & $1,801,220$ \\
\hline Otros Ingresos Operacionales & - & - \\
\hline Total de Ingresos Brutos & $1,817,782$ & $1,801,220$ \\
\hline Costo de Ventas (Operacionales) & $(1,331,592)$ & $(1,353,691)$ \\
\hline \multicolumn{3}{|l|}{ Otros costos operacionales } \\
\hline Total Costos Operacionales & $(1,331,592)$ & $(1,353,691)$ \\
\hline Utilidad Bruta & 486,190 & 447,529 \\
\hline Gastos de Ventas & $(208,985)$ & $(213,252)$ \\
\hline Gastos de Administración & $(84,947)$ & $(100,105)$ \\
\hline Provisión por pérdidas por desvalorización de activos & - & - \\
\hline Utilidad Operativa & 192,258 & 134,172 \\
\hline Ingresos Financieros & - & - \\
\hline Gastos Financieros & $(18,537)$ & $(11,382)$ \\
\hline Participación en los resultados de subsidiarias y afiliadas & $(13,543)$ & $(3,541)$ \\
\hline Ganancia o pérdida por instrumentos financieros derivados & (175) & $(2,505)$ \\
\hline Otros Ingresos & - & - \\
\hline Otros Gastos & $(19,363)$ & $(27,821)$ \\
\hline Efecto acumulado por cambios en las políticas contables & - & - \\
\hline Resultado por Exposición a la Inflación & - & 11,347 \\
\hline $\begin{array}{l}\text { Resultado antes de Gastos Extraordinarios, } \\
\text { Participaciones y del Impuesto a la Renta. }\end{array}$ & 140,640 & 100,270 \\
\hline Participación de los trabajadores corrientes y diferidos & $(14,368)$ & 406 \\
\hline Impuesto a la Renta corriente y diferido & $(38,794)$ & 1,096 \\
\hline Resultado antes de Gastos Extraordinarios. & 87,478 & 101,772 \\
\hline $\begin{array}{l}\text { Gastos Extraordinarios (neto de participaciones e impuesto } \\
\text { a la renta) }\end{array}$ & - & - \\
\hline Resultado antes de Interés Minoritario. & 87,478 & 101,772 \\
\hline Interés Minoritario & - & - \\
\hline Utilidad (Perdida) Neta del Ejercicio. & 87,478 & 101,772 \\
\hline Dividendos de acciones Preferentes & - & - \\
\hline $\begin{array}{l}\text { Utilidad (Pérdida) Neta atribuible a los accionistas no } \\
\text { preferentes. }\end{array}$ & 87,478 & 101,772 \\
\hline Utilidad (pérdida) básica por acción común & 0.111285 & 0.129469 \\
\hline Utilidad (pérdida) básica por acción de inversión & 0.111285 & 0.129469 \\
\hline Utilidad (pérdida) diluida por acción común & 0.098093 & 0.114121 \\
\hline Utilidad (pérdida) diluida por acción de inversión & 0.098093 & 0.114121 \\
\hline
\end{tabular}

rentabilidad superior a su costo, pues de no ser así es mejor trasladar los bienes utilizados a otra actividad.

\section{Objetivos}

- Aumentar el valor de las empresa y por lo tanto la riqueza de los propietarios. Incluye maximizar la utilidad con la mínima inversión y lograr el mínimo costo de capital.

- Trabajar con el mínimo riesgo (proporción equilibrada entre el endeudamiento y la inversión de los socios, entre las obligaciones financieras de corto plazo y largo plazo y cobertura de los diferentes riesgos de cambio de intereses del crédito y de los valores bursátiles.

- Disponer de niveles óptimos de liquidez.

\section{Ventajas}

- Mejor conocimiento de las unidades estratégicas de negocios (UEN) que crean o destruyen Valor Económico.

- Mantener un conocimiento permanente entre el mercado productivo y el mercado financiero a fin de conseguir el éxito empresarial deseado.

- Desarrollo de estrategias encaminadas a incrementar el valor futuro de la empresa

- Rediseño de procesos internos para alinearlos al concepto de creación de Valor.

\section{CÁLCULODELEVA}

Para esta parte se está considerando los Estados Financieros de Alicorp (ver cuadros 2 y 3 ), una de las empresa más importantes del medio en cuanto a la producción y comercialización de productos de consumo masivo de los años 2004 y 2005. 
Para determinar el EVA se ha considerado el desarrollo en 4 pasos como sigue:

\section{PASO 1:}

\section{IDENTIFICAR EL CAPITAL INVERTIDO EN} LA EMPRESA.

\begin{tabular}{|l|r|r|}
\hline Conceptos & \multicolumn{1}{|c|}{$\mathbf{2 0 0 5}$} & \multicolumn{1}{|c|}{$\mathbf{2 0 0 4}$} \\
\hline Cuentas por Cobrar Comerciales & 253,450 & 258,608 \\
\hline Existencias & 273,645 & 230,731 \\
\hline Otros activos corrientes & - & - \\
\hline Caja & 11,069 & 5,420 \\
\hline Total 1 & $\mathbf{5 3 8 , 1 6 4}$ & $\mathbf{4 9 4 , 7 5 9}$ \\
\hline Cuentas por pagar & 152,097 & 199,026 \\
\hline Otras cuentas por pagar & 68,451 & 39,983 \\
\hline Total 2 & $\mathbf{2 2 0 , 5 4 8}$ & $\mathbf{2 3 9 , 0 0 9}$ \\
\hline Capital de Trabajo (1+2) & $\mathbf{3 1 7 , 6 1 6}$ & $\mathbf{2 5 5 , 7 5 0}$ \\
\hline Activos Fijos Netos & $\mathbf{9 6 4 , 9 5 6}$ & $\mathbf{9 5 2 , 6 8 8}$ \\
\hline Capital Invertido & $\mathbf{1 , 2 8 2 , 5 7 2}$ & $\mathbf{1 , 2 0 8 , 4 3 8}$ \\
\hline
\end{tabular}

\section{PASO 2 : COSTO PROMEDIO PONDERADO (CPPC)}

Para hallar este valor se ha considerado la siguiente fórmula:

$\mathrm{CPPC}=\mathrm{Kd} . \times($ pasivo/activo $) \times(1-\mathrm{t})+K c \times($ patrimonio $/$ activo $)$

Para el cálculo del costo de oportunidad del patrimonio se considera que los accionistas requieren una rentabilidad sobre sus inversiones del $10 \%$ anual (Kc).

\begin{tabular}{|l|r|r|}
\hline Conceptos & $\mathbf{2 0 0 5}$ & $\mathbf{2 0 0 4}$ \\
\hline \% Deuda = Pasivos/Activos & $42.87 \%$ & $45.62 \%$ \\
\hline Costo de la deuda & $\mathbf{2 . 3 8 \%}$ & $\mathbf{1 . 4 2} \%$ \\
\hline Tasa Impuesto a la renta & $30 \%$ & $30 \%$ \\
\hline Costo de la deuda después de impuestos & $\mathbf{1 . 6 7 \%}$ & $\mathbf{0 . 9 9 \%}$ \\
\hline \% Patrimonio = Patrimonio/Activos & $57.13 \%$ & $54.38 \%$ \\
\hline Costo de Capital & $12.00 \%$ & $12.00 \%$ \\
\hline Costo Patrimonio & $\mathbf{6 . 8 6} \%$ & $\mathbf{6 . 5 3 \%}$ \\
\hline Costo Promedio Ponderado (CPPC) & $\mathbf{8 . 5 2} \%$ & $\mathbf{7 . 5 2} \%$ \\
\hline
\end{tabular}

Nota:

El CPPC depende del nivel del tipo de interés corriente (a mayores tasas de interés, el CPPC será más alto) y al tipo de negocio en la cual se desempeña la empresa (a mayor riesgo mayor CPPC).

\section{PASO 3: CÁLCULO DE UOII Y ROI}

\begin{tabular}{|l|r|r|}
\hline Conceptos & $\mathbf{2 0 0 5}$ & $\mathbf{2 0 0 4}$ \\
\hline Ventas & $1,817,782.00$ & $1,801,220.00$ \\
\hline Costo de Ventas & $(1,331,592.00)$ & $(1,353,691.00)$ \\
\hline Utilidad Bruta & $\mathbf{4 8 6 , 1 9 0 . 0 0}$ & $\mathbf{4 4 7 , 5 2 9 . 0 0}$ \\
\hline Gastos de Ventas & $(208,985.00)$ & $(213,252.00)$ \\
\hline Gastos de Administración & $(84,947.00)$ & $(100,105.00)$ \\
\hline Utilidad Operativa & $\mathbf{1 9 2 , 2 5 8 . 0 0}$ & $\mathbf{1 3 4 , 1 7 2 . 0 0}$ \\
\hline Participación Laboral & & \\
\hline Impuestos sobre EBIT & $(38,794.00)$ & $1,096.00$ \\
\hline UAll & $\mathbf{1 5 3 , 4 6 4 . 0 0}$ & $\mathbf{1 3 5 , 2 6 8 . 0 0}$ \\
\hline Capital Invertido & $\mathbf{1 , 2 8 2 , 5 7 2 . 0 0}$ & $\mathbf{1 , 2 0 8 , 4 3 8 . 0 0}$ \\
\hline ROI & $\mathbf{1 1 . 9 7 \%}$ & $\mathbf{1 1 . 1 9 \%}$ \\
\hline
\end{tabular}

PASO 4: CÁLCULO DEL EVA

\begin{tabular}{|l|r|r|}
\hline \multicolumn{1}{|c|}{ Conceptos } & $\mathbf{2 0 0 5}$ & $\mathbf{2 0 0 4}$ \\
\hline ROI & $11.97 \%$ & $11.19 \%$ \\
\hline CPPC & $7.38 \%$ & $6.43 \%$ \\
\hline ROI - CPPC & $4.59 \%$ & $4.76 \%$ \\
\hline Capital Invertido & $\mathbf{1 , 2 8 2 , 5 7 2 . 0 0}$ & $\mathbf{1 , 2 0 8 , 4 3 8 . 0 0}$ \\
\hline EVA = (ROI - CPPC) * Capital Invertido & $\mathbf{5 8 , 8 3 4 . 0 8}$ & $\mathbf{5 7 , 5 7 1 . 2 6}$ \\
\hline Incremento de EVA 2005 vs. 2004 & $2.19 \%$ & \\
\hline
\end{tabular}

\section{Nota}

Este es el cálculo del EVA para un año. Si una empresa calcula el EVA para un trimestre, entonces debería también calcular el monto que debe pagar por el costo de capital trimestralmente o por el período que desee.

\section{ANÁLISIS DEL CASO}

Del valor que se ha determinado se puede mencionar que en los años de evaluación se ha generado valor para los accionistas, notándose que este valor se ha incrementado en un $2.19 \%$ con respecto al año 2004. Si bien es cierto la exigencia de generación de rentabilidad sobre el capital invertido ha sido mayor $(6.13 \%)$, esto ha sido compensado debido a un incremento en las ventas en un $0.92 \%$ y una reducción de los costos $(1.63 \%)$ y gastos operativos $(6.20 \%)$.

De este análisis se puede determinar que el EVA no solo es un número sino va más allá de este, define una gestión coherente que va desde definir una buena proyección y ejecución de las ventas, una logística con factores inamovibles de negociación de calidad y precio y tener una organización comprometida en los objetivos de la empresa, por ejemplo generar compensaciones de acuerdo a las metas establecidas, todo este desarrollo de gestión es en forma paralela lo cual determina una búsqueda continua para la generación de valor.

\section{CONCLUSIONES}

Los ratios financieros de medición de valor no son lo suficientemente apropiados para medir la rentabilidad del capital debido a que sólo son cocientes entre diferentes cuentas que en ningún momento toman en consideración, por ejemplo el concepto de costo de capital.

EI EVA evalúa la empresa sin distorsiones contables, es decir libre de aplicación de normativa contable permisibles por lo establecido en la legislación tributaria. 
EI EVA determina la generación del valor del capital invertido en un determinado periodo.

Se debe tener presente que la diferencia del (ROI CPPC) es un valor determinante para que una empresa genere valor, de lo que se desprende la siguiente regla de decisión:

\begin{tabular}{|l|l|}
\hline Comparación & Resultado \\
\hline ROI $>$ CPPC & Genera valor \\
\hline ROI = CPPC & No crea ni destruye valor \\
\hline ROI < CPPC & Destruye valor \\
\hline
\end{tabular}

El EVA en la búsqueda de mejorar y/o mantener el valor de la empresa hace que la organización en general centren en conjunto que la realización sus actividades se realicen de forma eficiente $y$ económica. En esta búsqueda se debe establecer planeamiento, los métodos de evaluación y ante todo determinar recompensas para generar el compromiso con el personal es decir se debe implementar la gerencia basada en el valor.

\section{REFERENCIAS BIBLIOGRÁFICAS}

1. Amat Oriol (2000). EVA: Valor Económico Agregado. 1era. Edición. Editorial Gestión. Lima, Perú.

2. Andia Valencia, Walter (2004) Matemática Financiera y Evaluación de Proyectos. Editorial Centro de Investigación y Capacitación Empresarial. Lima, Perú.

3. Apaza Meza, Mario (2005). Contabilidad Estratégica. 1era. Edición. Editorial Editores.

4. Chu Rubio, Manuel (2005). Finanzas para no Financieros. 1era. Edición. Editorial Fondo Editorial UPC. LIma, Perú.

5. CONASEV. En:

www.conasev.gob.pe/emisoras/emi_eeff_menu.a sp.

(Visitado: 29-11-06). 